\title{
Selective culture medium failure in gonorrhoea
}

\author{
Sakina Rashid
}

This case report illustrates the need for regular review of culture methods and maintenance of thorough attempts at diagnosis prior to treatment of gonorrhoea. Vancomycin-sensitive gonococcal infection was discovered by repeated tests and by using a non-selective medium (locally). The support of the Gonococcus Reference Unit confirmed the findings. The observations are discussed in laboratory, treatment and control terms.

The use of selective media in the last quarter century has greatly facilitated the identification of gonorrhoea. How well any particular selective medium performs is likely to vary both from place to place and from time to time as gonococci of varying antibiotic sensitivities spread and circulate to and within individual countries.

One way of meeting this hazard is the use of selective and non-selective medium in harness. The cost-effectiveness of this is questionable. This case report shows how a small proportion of Neisseria gonorrhoeae failed to grow in selective media with implications in terms of diagnosis, management and control of the disease.

\section{Case report}

A 37 year old separated man attended the Department of Genito-Urinary Medicine in Sunderland with a four day history of urethral discharge and dysuria. His last sexual contact was with a known female one week prior to his attendance. He admitted to sexual contact with his wife four weeks before attending the department. On examination he was found to have a mucopurulent discharge. The first glass of urine was hazy with floccules and the second glass was clear. A gram stained smear showed intracellular gram-negative diplococci. Routine culture using a selective medium was negative. This was the fourth culture failure out of 26 cases confirmed by culture in the space of twelve months.

Formerly, a selective and non-selective medium has been used successfully for some 15-20 years. Confidence in the selected medium was high ${ }^{1}$ and routine use of a nonselective medium was abandoned a few years ago without any subsequent problem arising in diagnostic or test of cure routines. The selective medium used was modified Thayer Martin medium. It is prepared locally with an agar base with the addition of haemoglobin $10 \mathrm{~g} / \mathrm{l}$, nystatin 12,500 units/1, vancomycin $3 \cdot 0$ $\mathrm{mg} / \mathrm{l}$, colistin $7.5 \mathrm{mg} / \mathrm{l}$. The patient made a prompt response to treatment and was declared cured by negative smear and culture for gonorrhoea.

His consort attended a week after his smear, diagnosis and treatment. She was 35 years of age and separated. Her last sexual contact with the patient was eleven days prior to attendance. She also admitted to sexual contact with a regular partner of six weeks, nine days before attending the department. She was asymptomatic apart from mild vaginal pruritus. On clinical examination the only positive finding was muco-purulent vaginal discharge. Gram stained smears from urethra, cervix and rectal wall were negative for Gram negative diplococci, as were routine cultures for gonorrhoea from specimens from the urethra, cervix, rectum and pharynx on selective medium. These tests when repeated after 48 hours were also negative. With suspicions about the selective medium increasing the patient was again tested. The cervical smear only showed a few gram-negative intracellular diplococci. Cultures on the selective medium were again negative. Only the cervical culture on a non-selective modified Thayer Martin medium was positive. Also positive was the cervical specimen when duplicated culture specimens were submitted to a neighbouring laboratory using a modified selective New York City medium. This medium contained lincomycin $1.0 \mathrm{mg} / \mathrm{l}$, colistin sulphate 6.0 $\mathrm{mg} / \mathrm{l}$, amphotericin B $1.0 \mathrm{mg} / \mathrm{l}$, and trimethoprim lactate $6.5 \mathrm{mg} / \mathrm{l}$. Positive culture specimens were sent to the Gonococcus Reference Unit at Bristol where the sensitivity to vancomycin was confirmed.

\section{Discussion}

In his monograph on gonorrhoea, Morton $(1977)^{2}$ drew attention to warnings that some gonococci may be inhibited by vancomycin. Recommendations that the response may be reduction of its amount and/or the simultaneous use of a non-selective medium also was noted. As far back as 1973, Brorson et $a l^{3}$ claimed that, in their area, replacement of vancomycin with lincomycin increased the yield of positives by $10 \%$.

The question arises, is there a need to review the epidemiology of the distribution of sensitive and less sensitive gonococci within the UK? Has this changed with the $76 \%$ decline in the prevalence of gonorrhoea over the past decade? This report and the cluster of cases reported to Medical Society for the Study of Venereal Diseases in London on 13 January 1995 suggest a need for caution. ${ }^{4}$ Mild strains when prevalence rates are low are more threatening. One contributor suggested a nationwide survey of sensitivities of currently circulating gonococci be compared with the finding of 10-20 years ago.

There is also a need to discover if any change is being masked by the tendency to
Urinary Medicine, District General

Hospital, Kayll Road, Sunderland, SR4 7TP $S$ Rashid

Accepted for publication 16 January 1996 
confine diagnostic endeavours to a single test followed immediately in smear negative cases by epidemiological treatment. In the last three years there has been steady increase in the number of female patients treated epidemiologically for gonorrhoea (from 16.8 to $23 \cdot 6 \%) .5$

A combination of a growing, masked and unrecognised percentage of vancomycin sensitive gonococci, with a rise in epidemiological treatment and consequently inevitable truncating of the contact tracing process, could together be a threat to the control of gonorrhoea and the maintenance of current success. ${ }^{6}$

We suggest that the time may have come when future success will depend on a more thorough approach of the diagnosis before treatment. Has the time come for the United Kingdom first "Guidance Note"?

Grateful thanks are due to local colleagues for their laboratory support. The co-operation of Dr A E Jephcott, Gonococcus Reference Unit, Public Health Laboratory, Bristol, UK is much appreciated.

1 Taylor E, Phillips I. Assessment of selective medium for isolation of Neisseria gonorrhoea. Br $\mathcal{F}$ Venereal Dis 1979; 55:183-5.

2 Morton RS. Gonorrhoea, London: WB Saunders, 1977.

3 Brorson JE, Holmberg I, Nygren B, Seeberg S. Vancomycin sensitive strains of Neisseria gonorrhoea. A problem for the diagnostic laboratory. $B r \mathcal{F}$ Venereal $D i s$ 1973;49:452-3.

4 Brock G. A case cluster of Tissue Invasive Gonorrhoea. MSSVD Presentation 1995.

5 Department of Health-New Cases seen at NHS GenitoUrinary Medicine Clinic in England 1991, 1942, 1993 figures. Summary information from Form KC60.

6 Bignell C. The Eradication of Gonorrhoea. BMF. Editorial 1994;309:1 103-4. 